\title{
Formation and reduction of 5-hydroxymethylfurfural at frying temperature in model system as a function of amino acid and sugar composition
}

\begin{abstract}
5-Hydroxymethylfurfural (HMF) is formed during heat treatment of carbohydrate-containing foods, especially in a deep-fat frying process. This study aimed to investigate the effect of amino acids on the formation and reduction of HMF from glucose, fructose and sucrose at frying temperature in model systems containing binary mixtures of an amino acid and a sugar in equal concentrations $(0.3 \mathrm{M})$. The results revealed that the formation of HMF from sugars accelerated in the presence of acidic amino acids (i.e. glutamic and aspartic acids). Conversely, the presence of basic amino acids (i.e. lysine, arginine and histidine) led to reduced concentrations of HMF to non-detectable levels in model systems. The results showed that both $\mathrm{pH}$ and heating time significantly affected the formation of HMF from fructose in the presence of glutamic acid. In this regard, a higher amount of HMF was formed at lower $\mathrm{pH}$.
\end{abstract}

Keyword: 5-Hydroxymethylfurfural (HMF); Furanic compound; Deep-fat frying; Amino acid; Sugar; Model study 